\title{
Dynamical Velocity Selection: Marginal Stability
}

\author{
Wim van Saarloos \\ AT\& T Bell Laboratories, Murray Hill, New Jersey 07974 \\ (Received 1 April 1987)
}

\begin{abstract}
Dee and co-workers have advanced the idea that the natural velocity of fronts propagating into an unstable state is related to the stability of these fronts through "marginal stability." It is shown that this is indeed the case if front solutions lose stability through one particular mechanism. Marginal stability is derived for front propagation in the Swift-Hohenberg equation, and for an extension of the FisherKolmogorov equation, is only consistent with the existence of nonuniformly moving fronts in a certain range of parameters.

PACS numbers: $68.10 . \mathrm{La}, 03.40 . \mathrm{Kf}, 47.20 . \mathrm{Ky}$
\end{abstract}

In the last few years it has become appreciated in the physics community that the propagation of fronts into an unstable state forms a particularly interesting class of dynamical problems. ${ }^{1}$ Such fronts arise in such diverse fields as biology, ${ }^{2}$ combustion, ${ }^{3}$ nerve propagation, ${ }^{4}$ chemistry, ${ }^{5}$ and mathematics. ${ }^{6}$ In most examples studied, the states before and behind the front are rather featureless, and the fronts appear as wall-like excitations resembling propagating walls in liquid crystals. ${ }^{7}$ Physically richer examples of front propagation can be created, however, in fluid-flow instabilities when the system is suddenly brought above the threshold for a finitewavelength instability. ${ }^{8,9}$ In such experiments, the front propagation induces the wavelength selection of the state emerging behind it, thus leading to a form of dynamical pattern selection. It is the purpose of this paper to clearly identify the marginal-stability mechanism of front propagation advocated by Dee and co-workers, ' building on their ideas and those of Shraiman and Bensimon. ${ }^{10}$

The prototype equation exhibiting the simplest type of front propagation (without induced pattern selection) is the Fisher-Kolmogorov ${ }^{2}$ (FK) equation $\partial \phi / \partial t=\partial^{2} \phi / \partial x^{2}$ $+\phi-\phi^{3}$. The typical situation of interest here is the one in which a front is moving to the right, replacing the unstable state $\phi=0$ by the stable state $\phi=1$. What determines the velocity $v$ of a front growing out of a sufficiently localized region where $\phi \neq 0$ initially? This question is not answered by steady-state considerations, since the equation $\partial^{2} \phi / \partial x^{2}=-\imath \partial \phi / \partial x-\phi+\phi^{3}$ for uniformly translating fronts $\phi(x-\imath t)$ admits solutions for any velocity $v$ (as can easily be seen by exploiting the analogy with the equation of motion for a particle in a potential and subject to friction). That there is, nevertheless, some naturally selected velocity ${ }^{*}{ }^{*}$ was shown by Aronson and Weinberger, ${ }^{6}$ who rigorously proved that the speed of the physically most relevant fronts that are initially sufficiently localized [such that $\phi(x, t=0)$ drops off faster than $e^{-x}$ ], approaches the value $v^{*}=2$ for long times.

The result by Aronson and Weinberger ${ }^{6}$ strongly suggests that some sort of dynamical velocity-selection mechanism exists. This point of view was clearly advocated first by Dee and co-workers, ${ }^{1}$ who pointed out that the velocity $v^{*}=2$ of the FK equation is just the one at which the front appears to be "marginally stable," in that front solutions that move slower than $v^{*}$ are unstable to perturbations (in the co-moving frame), while those that move faster are stable. The marginal-stability hypothesis-i.e., the conjecture that the natural speed for propagation of initially localized fronts into an unstable state is in general the one corresponding to the marginal-stability point-was tested numerically by Dee and co-workers ${ }^{1}$ for several equations in which fronts give rise to dynamical pattern selection, e.g., the amplitude equation ${ }^{11}$ (AE) for complex $\phi$,

$$
\partial \phi / \partial t=\partial^{2} \phi / \partial x^{2}+\phi-|\phi|^{2} \phi
$$

and the Swift-Hohenberg ${ }^{12}(\mathrm{SH})$ for real $\phi$,

$$
\begin{array}{r}
\frac{\partial \phi}{\partial t}=-2 \frac{\partial^{2} \phi}{\partial x^{2}}-\frac{\partial^{2} \phi}{\partial x^{4}}+(\epsilon-1) \phi-\phi^{3}, \\
0<\epsilon<1 .
\end{array}
$$

These equations admit stable periodic states. In the numerical studies, ${ }^{1}$ the front speed was indeed found to approach the marginal-stability velocity.

Marginal stability can be tested experimentally in the Taylor-Couette ${ }^{8}$ and Rayleigh-Bénard ${ }^{9}$ flows, since just above the onset for instability these are described by the $\mathrm{AE}$ and $\mathrm{SH}$ equations. ${ }^{11,12}$ In the Taylor-Couette instability, the velocity of fronts was found ${ }^{8}$ to be a factor of 2 smaller than predicted by the theory. This discrepancy is as yet unresolved. ${ }^{13}$ Recent results ${ }^{9}$ on front propagation just above the Rayleigh-Bénard instability, however, are in excellent quantitative agreement with the theoretical prediction.

Clearly, for front propagation into an unstable state, marginal stability emerges as a viable dynamical velocity-selection mechanism with important practical and conceptual implications. In this paper I extend the ideas of Dee and co-workers ${ }^{1}$ and of Shraiman and Bensi- 
mon, ${ }^{10}$ so as to manifest the mechanism that can drive the front velocity to the marginal-stability value. I find that this happens when the front solutions lose stability because the group velocity for perturbations becomes larger than the envelope velocity. In this case, a Burgers-type equation ${ }^{14}$ for the local front structure drives the speed of (initially localized) fronts towards the marginal-stability velocity $\iota^{*}$. The marginal-stability scenario is shown not to apply if the steady-state solutions lose stability because of another mechanism, and this occurs in an extension of the FK equation. For the $\mathrm{AE}$ and $\mathrm{SH}$ equations, however, the marginal-stability point is attractive, as found empirically by Dee and coworkers. ${ }^{1}$

I first give an intuitive explanation for the seemingly counterintuitive result that natural front velocity is the slowest one at which a profile is stable. In passing, we note that such an effect is well known for crystal growth: If different facets of a crystal have different growth rates as in Fig. 1(a), the growth of the crystal becomes progressively dominated by the slowest facet. This can be viewed as a simple example of a dynamical selection mechanism. An important property of the type of fronts we are interested in here is that there is a branch of (stable) solutions whose velocity is increasing with the width of the profile (or its envelope). Figure l(b) illustrates this for two profiles growing into an unstable state. If their local growth rate is (about) the same, we see that for geometrical reasons the steeper profile has the slowest velocity; thus the velocity is an increasing function of the width. Consider now the profile of Fig. 1(c), which consists of two parts with different steepness and corresponding speeds. Clearly, the slowest-moving part (full line) expands at the expense of the faster part
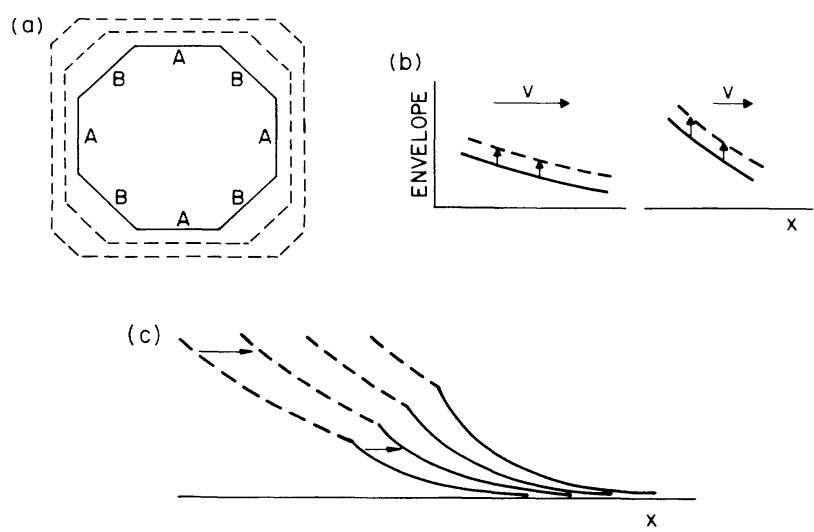

FIG. 1. (a) The growth of a crystal, indicated by the dashed lines, becomes gradually more dominated by the growth of the slowest facet. (b) Illustration of the fact that the velocity decreases with the steepness of the profile. (c) For a profile consisting of two parts moving with different speeds roughly proportional to their width, the crossover point moves up in time, so that the fast part "retreats" from the leading edge. (dashed line), and increasingly dominates the appearance of the front. This velocity-selection mechanism is an immediate consequence of the fact that the fastermoving portion effectively decreases the width of the profile and hence its speed. Of course, the discontinuities in slope of Fig. 1(c) do not occur for the smooth profiles relevant for Eqs. (1) and (2), but we shall see that essentially the same dynamical mechanism can drive $r$ towards the marginal-stability value $\iota^{*}$ in those cases.

If we consider instead of Fig. 1(c) a profile whose asymptotic (large- $x$ ) behavior is given by the slowerdecaying dashed portion, because the initial conditions are not sufficiently localized, this faster-moving portion actually expands in time and dominates the long-time behavior. Analogously, the marginal-stability point is only approached for sufficiently localized initial conditions, and this was indeed found by Aronson and Weinberger $^{6}$ for the FK equation.

I now support the above discussion by an analysis in the leading edge of the profiles that extends work by Shraiman and Bensimon ${ }^{10}$ on first-order partial-differential equations. The analysis will be quite general for propagation into an unstable state $\phi=0$ described by an equation $\phi_{t}=F\left(\phi, \phi_{x}, \ldots\right)$, but I will illustrate the arguments by specifying to the $\mathrm{AE}$ and $\mathrm{SH}$ equations. (I use the $\mathrm{AE}$ as an example to stress that the discussion applies to equations that allow periodic states as well.) It is convenient ${ }^{10}$ to transform to the variable $u$ by writing $\phi=e^{-u}$, where I allow $u$ to be complex since for the $\mathrm{AE}$ and $\mathrm{SH}$ equation $\phi$ is oscillatory. In the leading edge, where $u^{r}(\equiv \operatorname{Re} u) \rightarrow \infty$ for $x \rightarrow \infty$, the dynamical equation for $u$ then becomes of the form

$$
u_{t}=-f\left(q, q_{x}, \ldots\right) \text {, }
$$

where $q \equiv u_{x}$. For the $\mathrm{AE}$ we have, e.g., $f\left(q, q_{x}\right)=1$ $+q^{2}-q_{x}$, and for the $\mathrm{SH}$ equation

$$
\begin{aligned}
f\left(q, q_{x}, q_{x x}, q_{x x x}\right)=\epsilon-1-2 q^{2} & -q^{4}+2 q_{x}\left(1+3 q^{2}\right) \\
& -3 q_{x}^{2}-4 q q_{x x}+q_{x x x} .
\end{aligned}
$$

Note that when $q=k$, independent of $x$, we have $f(q=k, 0,0, \ldots)=\omega(k)$, where $\omega(k)$ is given by the dispersion relation for perturbations of the form $\phi \sim e^{-k x+\omega t}$ [for the AE equation we have, e.g., $\omega(k)$ $\left.=1+k^{2}\right]$.

In a frame moving with a constant velocity $i$, (3) becomes $u_{t}=\imath q-f\left(q, q_{x}, \ldots\right)$. Let us first consider steady-state front solutions, i.e., a solution $q=k$ (=const) whose envelope propagates with a speed $v$. For such a solution, $\operatorname{Re} u_{t}=0$ in the moving frame, and thus we get $\operatorname{Re}[\imath k-f(k)]=0$, or

$$
v^{\prime}(k)=\operatorname{Re} \omega(k) / \operatorname{Re} k .
$$

To study the stability of these solutions, let us consider a small bounded perturbation $\delta \sim e^{-\mu x}$ in $u$, with $\operatorname{Re} \mu>0$. From the above equation for $u_{t}$ in the moving frame, we 
then find that perturbations with small $|\mu|$ are stable if $\operatorname{Re}\left[\left\{v(k)-f_{q}(q=k)\right\} \mu\right]>0$ for arbitrary small $\mu$ with $\operatorname{Re} \mu>0$. Since $f(q=k)=\omega(k)$, this inequality is obeyed, provided that

$$
\operatorname{Im}(d \omega / d k)=0 ; \quad v(k)>\operatorname{Re}(d \omega / d k) .
$$

The first part expresses that only those profiles whose wavelength $\lambda=2 \pi / \mathrm{Im} k$ (for given $\mathrm{Re} k$ ) is the most unstable one are insensitive to small perturbations [clearly, $\operatorname{Im}(d \omega / d k)=0$ is a necessary but not a sufficient condition; the necessary conditions and the stability to arbitrary wavelength perturbations are discussed later]. To understand the second part, note that $\operatorname{Re}(d \omega / d k)$ plays the role of the group velocity ${ }^{15}$ with which a local disturbance moves. So, when viewed in the co-moving frame, a disturbance moves to the left for $v>\operatorname{Re}(d \omega / d k)$ : The profile is stable because disturbances retreat from the leading edge in much the same way as the break point in Fig. 1 (c) retreats!

All stable solutions will at least have to satisfy the condition $\operatorname{Im}(d \omega / d k)=0$. Using this equation to express $\operatorname{Im} k$ as a function of $k^{r} \equiv \mathrm{Re} k$, we can write the velocity of these solutions as a function of $k^{r}$ only. The resulting functions $i \cdot\left(k^{r}\right)$ for the $\mathrm{AE}$ and $\mathrm{SH}$ equations are depicted in Fig. 2(a). Note that $v$ diverges for $k^{r} \rightarrow 0$ and, according to Fig. 1 (b), this is a general feature of the solution for front propagation into an unstable state. Moreover, the second condition in (4) shows that these solutions are stable to long-wavelength perturbations because the group velocity is smaller than the envelope velocity. The marginal-stability point $k=k^{*}, v=v^{*}$, where the latter effect ceases to ensure stability, is, according to (5), given by ${ }^{\prime}$

$$
\left.\operatorname{Im} \frac{d \omega}{d k}\right|_{k=k^{*}}=0, \quad \iota^{*}=\left.\frac{d \omega}{d k}\right|_{k=k^{*}},
$$

with $\iota^{*}$ given by (4). It is straightforward to show that these equations precisely determine the extrema of the branch $v\left(k^{r}\right)$ given by $\operatorname{Im}(d \omega / d k)=0$; they are indicated by dots in Fig. 2(a). Taken together, these results therefore demonstrate that there often is a branch $v^{\prime}\left(k^{r}\right)$ of stable-front solutions for small $k^{r}$, at the bottom of which lies the marginal-stability point.

To understand how the speed of a front solution develops, let us consider profiles whose envelopes are monotonically decreasing. It is then useful ${ }^{10}$ to write an equation for the evolution of $q$ in terms of the variables $u^{r}$ and $t$, since $u^{r}$ moves with the profile. Using that

$$
\frac{\partial}{\partial u^{r}} \frac{\partial u(x, t)}{\partial t}=\left(q^{r}\right)^{-1}\left[\left.\frac{\partial q}{\partial t}\right|_{u}-f^{r} q_{u}\right],
$$

where a subscript $u$ denotes differentiation with respect to $u^{r}$, we obtain upon differentiation of Eq. (3)

$$
q_{t}=\left[f^{r}-f_{q} q^{r}\right] q_{u}+\mathcal{L} q,
$$
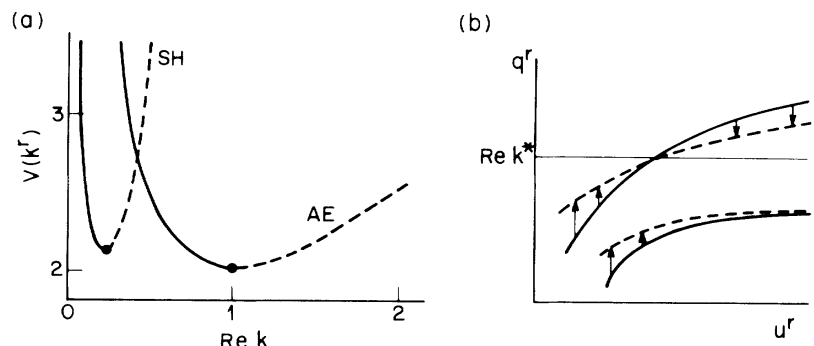

FIG. 2. (a) $v$ as a function of $\mathrm{Re} k$ for solutions of the $\mathrm{AE}$ and SH equations (for $\epsilon=\frac{1}{4}$ ), satisfying $\operatorname{Im}(d \omega / d k)=0$. (b) Qualitative sketch of the dynamical behavior of $q^{r}$ for two different initial conditions. The initial $q^{r}$ is drawn with a solid line and the one at a later time with a dashed line.

with $\mathcal{L} q=-f_{q_{u}} q_{u u}-f_{q_{u u}} q_{u u u}-\cdots$. Although $q=k$ is a solution of Eq. (7), we recognize in the term between square brackets for $q=k$ the combination $k^{r}\left[v(k)-f_{q}\right]$ $=k^{r}[l \cdot(k)-d \omega / d k]$ that according to (5) determines the stability of solutions. Therefore, the marginal-stability point where this term vanishes corresponds to a special fixed point of this equation, and the relevant nonlinearities for velocity selection are in the first term on the right-hand side of (7), provided that the operator $\mathcal{L}$ is stable. To illustrate this, consider the case in which the highest derivative in $L$ is of second order, as is the case for the $\mathrm{AE}$ equation. We can then approximate $\angle q=D q_{u u}$ with $D=-f_{q_{u}}(=\operatorname{Re} k>0$ for the AE equation), and consider the term between square brackets as a function of $q$ only, so that (7) reduces to $q_{t}$ $=c(q) q_{u}+D q_{u u}$, with $c(q)$ real in view of (5). This is of the form of the well-known Burgers equation, ${ }^{14}$ for which it is straightforward to show that the nonlinear term indeed drives $q$ to the marginal-stability value for sufficiently localized initial conditions. More generally, let us for the moment concentrate on the first term in Eq. (7), so that $q_{t}=\left[f^{r}-q^{r} f_{q}\right] q_{u}$, where we approximate $f$ as a function of $q$ only. In view of the above analysis, the term between square brackets is positive for small $q^{r}$ along the stable branch and vanishes at $q=k^{*}$. Thus, upon writing $q\left(u^{r}, t\right)=k^{*}+\rho\left(u^{r}, t\right)$, we get to lowest nontrivial order $\rho_{t}=-c \rho \rho_{u}$ with $c$ a positive constant. In the most important case in which the initial profile is sufficiently localized, i.e., drops off faster than $\exp \left[-\left(\operatorname{Re} k^{*}\right) x\right], q^{r}$ is larger than $\operatorname{Re} k^{*}$ for large $u^{r}$, as sketched in Fig. 2(b). Since $\rho_{u}$ is positive in this case $\rho$ decays according to the above equation; in other words, $q^{r}$ approaches $\operatorname{Re} k^{*}$ for all $u^{r}$, and by implication the front velocity $v$ approaches $v^{*}$. A case in which the initial conditions fall off less fast than $\exp \left[-\left(\operatorname{Re} k^{*}\right) x\right]$ is also depicted in Fig. 2(b). As indicated, $q^{r}$ then stays smaller than $\operatorname{Re} k^{*}$ at later times, and as a result the speed of the profile will approach a value larger than $v^{*}$. Thus, provided that the operator $\mathcal{L}$ is stable, we see that the first term in (7) both governs the stability of fronts 
and drives the velocity to the marginal-stability value. This is the mechanism illustrated in Fig. 1. Within the context of this approach, these considerations therefore show that the results derived by Aronson and Weinberger for the FK equation can indeed be generalized to a large class of equations describing front propagation into an unstable state. For a specific equation, however, the condition under which the first term in (7) drives $v$ towards $l^{*}$ is the requirement that the operator $\mathcal{L}$ be stable on the branch of solutions obeying (5). For the $\mathrm{AE}$ equation this is indeed the case, as follows from the earlier observation that $\mathcal{L} q=D q_{u u}$ (with $D=-f_{q_{u}}$ $=k^{r}$ ), so that Eq. (7) reduces to a Burgers-type equation $^{14}$ from which the same conclusions are reached as above. In a more detailed paper ${ }^{7}$ I will show that the eigenmodes of $\mathcal{L}$ for the $\mathrm{SH}$ equation are indeed stable and hence, together with the above analysis, that the marginal-stability point of this equation is attractive. This provides an a posteriori justification for the numerical observations of Dee and co-workers. ${ }^{1}$

If front solutions first lose stability because the eigenmodes of $\mathcal{L}$ become unstable, "marginal stability" does not hold. That the requirement that $\mathcal{L}$ be stable is a nontrivial condition is shown by the extended FK equation $\phi_{t}=\phi_{x x}-\gamma \phi_{x x x x}+\phi-\phi^{3}$. For $\gamma<\frac{1}{12}$, the marginal-stability point is attractive, and the front velocity will approach $v^{*}$. For $\gamma>\frac{1}{12}$, however, the eigenmodes of $\mathcal{L}$ of uniformly translating solutions $\phi(x-v t)$ go unstable first, so that marginal stability cannot hold for these type of fronts; for $\gamma>\frac{1}{12}$, marginal stability can therefore only apply to nonuniformly moving fronts whose dynamics do not correspond to a simple translation of the profile as a whole. This is presently being tested by Dee.

An important underlying assumption of the present formulation is that there is a continuous branch of stable steady-state solutions, since only then is the dynamics not constrained by strong nonlinearities behind the leading edge. For the FK equation, the particle-on-the-hill analogy demonstrates the existence of this branch of stable solutions. This analogy is specific to the FK equation, but a counting argument ${ }^{7}$ demonstrates that the existence of a continuum family of stable fronts is a general feature of uniformly moving fronts $\phi(x-v t)$ propagating into a steady state. Furthermore, Collet and Eckmann ${ }^{14}$ have recently shown that the $\mathrm{SH}$ equation admits (for small $\epsilon$ ) a two-parameter family of front solutions as a result of the additional freedom introduced by the wavelength $\lambda$ of the pattern emerging behind the front. This is likely to occur in general ${ }^{7}$ for fronts lead- ing to periodic states. Clearly, most of these front solutions of the $\mathrm{SH}$ equation will be unstable, since there is no reason to expect that $\operatorname{Im}(d \omega / d k)=0$ for an arbitrary solution. As a result, the solutions that are stable will form only a one-parameter continuous subset of these, which is just what one expects to be necessary for the leading-edge analysis to work. These questions as well as a number of open problems will be discussed in a more detailed account, ${ }^{7}$ in which I will also generalize some other observations by Dee and co-workers ${ }^{1}$ and discuss important differences between solutions whose envelope is moving with a constant velocity and solutions $\phi(x-\imath t)$ that correspond to a uniform translation of the profile as a whole.

I am grateful to Pierre C. Hohenberg and G. Dee for helpful discussions.

${ }^{1}$ G. Dee and J. S. Langer, Phys. Rev. Lett. 50, 383 (1983); E. Ben-Jacob, H. R. Brand, G. Dee, L. Kramer, and J. S. Langer, Physica (Amsterdam) 14D, 348 (1985); G. Dee, J. Stat. Phys. 39, 705 (1985); see also J. S. Langer and H. Müller-Krumbhaar, Phys. Rev. A 27, 499 (1983).

${ }^{2}$ R. A. Fisher, Ann. Eugenics 7, 355 (1937); A. Kolmogorov, I. Petrovsky, and N. Piskunov, Bull. Univ. Moskou Ser. Int. Sec. A 1, (No. 6), 1 (1937).

${ }^{3}$ I. M. Gel'fand, Usp. Mat. Nauk 14, 87 (1959) [Russ. Math. Surv. 29, 295 (1963)].

${ }^{4}$ A. C. Scott, Neurophysics (Wiley, New York, 1977).

${ }^{5}$ See, e.g., P. Fife, in Mathematical Aspects of Reacting and Diffusing Systems, edited by S. Levin, Lecture Notes in Biomathematics Vol. 28 (Springer-Verlag, New York, 1979).

${ }^{6}$ D. G. Aronson and H. F. Weinberger, Adv. Math. 30, 33 (1978).

${ }^{7}$ See, for further discussion, W. van Saarloos, to be published.

${ }^{8}$ G. Ahlers and D. S. Cannell, Phys. Rev. Lett. 50, 1583 (1983).

${ }^{9}$ J. Fineberg and V. Steinberg, Phys. Rev. Lett. 58, 1332 (1987).

${ }^{10}$ B. Shraiman and D. Bensimon, Phys. Scr. T9, 123 (1985)

${ }^{11}$ A. C. Newell and J. A. Whitehead, J. Fluid Mech. 38, 279 (1969).

12J. Swift and P. C. Hohenberg, Phys. Rev. A 15, 319 (1977).

${ }^{13}$ M. Lücke, M. Mihelcic, and K. Wingerath, Phys. Rev. Lett. 52, 625 (1984); Phys. Rev. A 31, 396 (1985).

${ }^{14}$ P. Collet and J. P. Eckmann, Commun. Math. Phys. 107, 39 (1986), and Physica (Amsterdam) 140A, 96 (1986).

${ }^{15}$ See, e.g., G. B. Whitham, Linear and Nonlinear Waves (Wiley, New York, 1974). 